\title{
Coefficient of Variation Based Image Selective Segmentation Model Using Active Contours
}

\author{
Noor Badshah ${ }^{1}$, Ke Chen ${ }^{2, *}$, Haider $\mathrm{Ali}^{1}$ and Ghulam Murtaza ${ }^{1}$ \\ ${ }^{1}$ Department of Basic Sciences, UET Peshawar, Pakistan. \\ ${ }^{2}$ Centre for Mathematical Imaging Techniques and Department of \\ Mathematical Sciences, The University of Liverpool, United Kingdom.
}

Received 9 March 2012; Accepted (in revised version) 19 April 2012

Available online 27 April 2012

\begin{abstract}
Most image segmentation techniques efficiently segment images with prominent edges, but are less efficient for some images with low frequencies and overlapping regions of homogeneous intensities. A recently proposed selective segmentation model often works well, but not for such challenging images. In this paper, we introduce a new model using the coefficient of variation as a fidelity term, and our test results show it performs much better in these challenging cases.
\end{abstract}

AMS subject classifications: 68U10, 62G30

Key words: Segmentation, Coefficient of Variation (CoV), level set, functional minimisiation, Total Variation (TV).

\section{Introduction}

In image segmentation, the main issue is to extract features according to a given criterion $[3,5,6,10,11,13,15]$. There are two important method categories. The first category refers to edge-based methods, where active contours have proven their effectiveness $[5,8,13,20]$. The general idea behind an active contours model is to apply partial differential equations (PDEs) to deform a curve towards the boundaries of the object of interest, so the contour is driven towards image edges. For edge detection, most models use an edge detector function which depends on the gradient of a given image $[3,10,11]$. The second category contains region-based methods, including active contour models involving minimum description length criteria [14], region growing and emerging [2], MumfordShah functional minimisation [18] and watershed algorithms [24] as examples.

${ }^{*}$ Corresponding author. Email addresses: noor2knoor@googlemail.com (N. Badshah), k.chen@ liv.ac.uk (K. Chen) 
Let $z(x, y)$ be a given image defined on a rectangular domain $\Omega$. Mumford and Shah (MS) [18] proposed the general model

$$
\min _{u, \Gamma} F(u, \Gamma)=\mu . \text { length }(\Gamma)+\lambda \int_{\Omega}|z-u|^{2} d x+\int_{\Omega \mid \Gamma}|\nabla u|^{2} d x
$$

to automatically find the edge $\Gamma$ of $z$ by a piecewise smooth function $u$. The Chan-Vese (CV) [6] model is a special case of the piecewise constant MS model when restricted to only two phases. Since the CV model is not based on the gradient of the image $z(x, y)$ for the stopping process, it can detect contours both with and without gradients. The CV active contour model uses the energy minimisation functional given by:

$F\left(c_{1}, c_{2}, \Gamma\right)=\mu$. length $(\Gamma)+\lambda_{1} \int_{\operatorname{inside}(\Gamma)}\left|z-c_{1}\right|^{2} d x d y+\lambda_{2} \int_{\text {outside }(\Gamma)}\left|z-c_{2}\right|^{2} d x d y$

where $z$ is a given image, $\Gamma$ is an unknown boundary, and $c_{1}$ and $c_{2}$ are constants that depend on $\Gamma$ and represent the average value of $z$ inside and outside of $\Gamma$ respectively.

The above categories of segmentation models are global, because all global features are to be segmented. Although useful, in certain segmentation problems we need to segment a particular object and not all objects. Selective segmentation is a task in which an object or region of interest is detected, given additional information of geometric constraints in the form of list of points near the object or region.

Based on the work of Refs. $[6,10,11]$, we recently proposed a mixed model of edgebased and region-based methods that provved more robust for noisy images [3]. However, this model can produce spurious objects - i.e. fails the selection in some cases. Now we equip our model with a new type of fidelity term for it to perform better, even when edges are not prominent or an image has overlapping regions with almost homogeneous intensities. The fidelity term is based on a coefficient of variation, and our experimental results demonstrate the superior performance of this new model.

This paper is organised in the following way. A review of the previous model [3] is presented in Section 2. Our new model of minimisation and the Euler-Lagrange equation are discussed in Section 3. We describe a semi-implicit method and an additive operator splitting (AOS) method for solving the PDE on Section 4, and give some experimental results in Section 5.

\section{BC Model}

To segment a given image $z$ or find the boundary $\Gamma$ of a desirable feature, the recent Badshah and Chen (BC) model [3] solves

$$
\min _{c_{1}, c_{2}, \Gamma} F\left(\Gamma, c_{1}, c_{2}\right)
$$


where

$$
\begin{aligned}
F\left(\Gamma, c_{1}, c_{2}\right)= & \mu \int_{\Gamma} d(x, y) g(|\nabla z|) d s \\
& +\lambda_{1} \int_{\text {outside }(\Gamma)}\left(z-c_{1}\right)^{2} d x d y+\lambda_{2} \int_{\text {inside }(\Gamma)}\left(z-c_{2}\right)^{2} d x d y
\end{aligned}
$$

and $\mu, \lambda_{1}$ and $\lambda_{2}$ are constants and are used for assigning different weights, and $c_{1}$ and $c_{2}$ are the mean intensities outside and inside a contour $\Gamma$ respectively. The distance function is [11]

$$
d(x, y)=\prod_{i=1}^{m}\left(1-e^{-\frac{\left(x-x_{i}\right)^{2}}{2 \sigma^{2}}} e^{-\frac{\left(y-y_{i}\right)^{2}}{2 \sigma^{2}}}\right), \quad \forall(x, y) \in \Omega,
$$

where the marker set

$$
\mathscr{A}=\left\{\left(x_{i}, y_{i}\right): i=1,2,3, \cdots, m\right\}
$$

are the given geometrical constraints and we wish to detect the boundary of an interested object near $\mathscr{A}$. Intuitively, it is clear that $d \approx 0$ in the neighbourhood of $\mathscr{A}$.

The function $g(|\nabla z|)$ is called as an edge detector function, and a popular choice is

$$
g(|\nabla z|)=\frac{1}{1+|\nabla z|^{2}}
$$

although there are many other choices. Since the edge is the portion of an image where there is a sudden change in the intensity function, the value of the function $|\nabla z|^{2}$ is large at the pixels belonging to the edge. Consequently, $g(|\nabla z|) \simeq 0$ near an edge.

The first term of the BC model is $\int_{\Gamma} d(x, y) g(|\nabla z|) d s$, similar to Refs. [10,11]. The aim is to find the unknown boundary curve $\Gamma$ by minimising their proposed functional. Since this model depends on an edge detector function using gradient information, this causes model malfunction with noisy images or images with fuzzy or discrete edges. Isotropic Gaussian smoothing can be used to smooth z, but unfortunately it also smoothrs the edges, geodesic active contours alone are not sufficient. To empower this model to work with noisy images, region information is also used - viz. by adding $\lambda_{1} \int_{\text {outside }(\Gamma)}(z-$ $\left.c_{1}\right)^{2} d x d y+\lambda_{2} \int_{\text {inside }(\Gamma)}\left(z-c_{2}\right)^{2} d x d y$ with the edge information, to form the remaining two terms of the BC model that also appear in the CV model [6]. In this way, the CV model advantages are utilised in the BC model.

The application of a level set formulation $[19,20,22]$ enables implicit representation of the boundary, interior and exterior regions of the object of interest in a given image. Let us denote the exterior of $\Gamma$ by $\Omega^{+}$and the interior by $\Omega^{-}$. If $\phi: \Omega \rightarrow \mathbb{R}$, a Lipchitz continuous function is a level set function such that

$$
\begin{aligned}
& \Gamma=\{(x, y): \phi(x, y)=0\}, \\
& \left.\Omega^{+}=\{x, y): \phi(x, y)>0\right\}, \quad \Omega^{-}=\{(x, y): \phi(x, y)<0\} .
\end{aligned}
$$


Further, the quantities in Eq. (2.1) can be reformulated as

$$
\begin{aligned}
& \text { length }\{\Gamma\}=\int_{\Omega}|\nabla H(\phi)|=\int_{\Omega} \delta(\phi)|\nabla \phi| d x d y, \\
& \int_{\text {outside }(\Gamma)}\left|z-c_{1}\right|^{2} d x=\int_{\Omega}\left|z-c_{1}\right|^{2} H(\phi) d x d y, \\
& \int_{\text {inside }(\Gamma)}\left|z-c_{2}\right|^{2} d x=\int_{\Omega}\left|z-c_{2}\right|^{2}(1-H(\phi)) d x d y,
\end{aligned}
$$

where the one-dimensional Heaviside and Dirac delta functions

$$
H(x)=\left\{\begin{array}{ll}
1 & \text { if } x \geq 0 \\
0 & \text { if } x<0
\end{array} \quad \text { and } \quad \delta(x)=H^{\prime}(x)\right.
$$

will be respectively replaced by regularised versions $[6,7,19]$

$$
H_{\epsilon}(w)=\frac{1}{2}\left(1+\frac{2}{\pi} \arctan \left(\frac{w}{\epsilon}\right)\right), \quad \delta_{\epsilon}(w)=H_{\epsilon}^{\prime}(w)=\frac{\epsilon}{\pi\left(\epsilon^{2}+w^{2}\right)} .
$$

Thus Eq. (2.1) becomes

$$
\begin{aligned}
F_{\epsilon}\left(\phi, c_{1}, c_{2}\right)= & \mu \int_{\Omega} d(x, y) g(|\nabla z|) \delta_{\epsilon}(\phi)|\nabla \phi| d x d y+\lambda_{1} \int_{\Omega}\left|z(x, y)-c_{1}\right|^{2} H_{\epsilon}(\phi) d x d y \\
& +\lambda_{2} \int_{\Omega}\left|z(x, y)-c_{2}\right|^{2}\left(1-H_{\epsilon}(\phi)\right) d x d y .
\end{aligned}
$$

Keeping $\phi$ fixed and minimising $F_{\epsilon}\left(\phi, c_{1}, c_{2}\right)$ with respect to $c_{1}$ and $c_{2}$, we have

$$
c_{1}(\phi)=\frac{\int_{\Omega} z(x, y) H_{\epsilon}(\phi) d x d y}{\int_{\Omega} H_{\epsilon}(\phi) d x d y} \quad \text { and } \quad c_{2}(\phi)=\frac{\int_{\Omega} z(x, y)\left(1-H_{\epsilon}(\phi)\right) d x d y}{\int_{\Omega}\left(1-H_{\epsilon}(\phi)\right) d x d y}
$$

assuming that the curve has a non-empty exterior and non-empty interior in $\Omega$. Now keeping $c_{1}, c_{2}$ fixed and minimising $F_{\epsilon}$ with respect to $\phi$ yields the following Euler-Lagrange equation for $\phi$ :

$$
\begin{cases}\delta_{\epsilon}(\phi)\left[\mu \operatorname{div}\left(G(x, y) \frac{\nabla \phi}{|\nabla \phi|}\right)\right. & \\ \left.-\lambda_{1}\left(z(x, y)-c_{1}\right)^{2}+\lambda_{2}\left(z(x, y)-c_{2}\right)^{2}\right]=0 & \text { in } \Omega, \\ \frac{G(x, y) \delta_{\epsilon}(\phi)}{|\nabla \phi|} \frac{\partial \phi}{\partial \vec{n}}=0 & \text { on } \partial \Omega,\end{cases}
$$

where $G(x, y)=d(x, y) g(|\nabla z|), \vec{n}$ is the unit exterior normal to the boundary $\partial \Omega$, and $\partial \phi / \partial \vec{n}$ is the normal derivative of $\phi$ at the boundary. 
The above PDE may be considered as a steady state form of the evolution equation

$$
\frac{\partial \phi}{\partial t}=\delta_{\epsilon}(\phi)\left[\mu \nabla \cdot\left(G(x, y) \frac{\nabla \phi}{|\nabla \phi|}\right)-\lambda_{1}\left(z-c_{1}\right)^{2}+\lambda_{2}\left(z-c_{2}\right)^{2}\right] \text { in } \Omega,
$$

where $\phi(t, x, y)=\phi_{0}(x, y)$ in $\Omega$. For robustness and iteration initialisiation, a balloon term $\alpha G(x, y)|\nabla \phi|$ was added, where $\alpha$ is constant [11]. To solve the above evolution equation, an additive operator splitting method (AOS) was used $[16,25]$.

Although we have shown in Ref. [3] that the BC model is more robust than previous models, there are images where it fails - in particular, MRI and CT images with fuzzy edges, unilluminated organs and overlapping homogeneous regions. Since the BC model (2.1) involves the fidelity term or region detector $\int\left|z-c_{1}\right|^{2} d x d y+\int\left|z-c_{2}\right|^{2} d x d y$ taken from the CV model [6], not only are its advantages but also its weaknesses are carried to the BC model. Experimental results showed that the BC model does not work efficiently for some images, due to the detection of spurious objects. Better region detectors are required for the detection of low contrast, unilluminated and overlapping homogeneous regions.

\section{Coefficient of Variation Equipped Selective Model}

Based on the concept of coefficient of variation ( $\mathrm{CoV})$ [1,17], we introduce a new type of fidelity term — viz.

$$
\lambda_{1} \int_{\text {outside }(\Gamma)} \frac{\left(z-c_{1}\right)^{2}}{c_{1}^{2}} d x d y+\lambda_{2} \int_{\text {inside }(\Gamma)} \frac{\left(z-c_{2}\right)^{2}}{c_{2}^{2}} d x d y .
$$

Thus we propose the following model, to be denoted by CSM:

$$
\min _{\Gamma, c_{1}, c_{2}} F\left(\Gamma, c_{1}, c_{2}\right)
$$

where

$$
\begin{aligned}
F\left(\Gamma, c_{1}, c_{2}\right)= & \mu \int_{\Gamma} d(x, y) g(|\nabla z|) d s+\lambda_{1} \int_{\operatorname{outside}(\Gamma)} \frac{\left(z-c_{1}\right)^{2}}{c_{1}^{2}} d x d y \\
& +\lambda_{2} \int_{\text {inside }(\Gamma)} \frac{\left(z-c_{2}\right)^{2}}{c_{2}^{2}} d x d y
\end{aligned}
$$

and $\mu, \lambda_{1}, \lambda_{2}$ are constants and used for assigning different weights. For a discrete image $z$, the new model may be explained as follows.

The variance defined by

$$
\operatorname{Var}(z)=\frac{1}{N} \sum_{i, j}\left(z_{i, j}-\operatorname{Mean}(z)\right)^{2}
$$


where $z_{i, j}$ denotes the image intensity at position $(i, j)$ and $\operatorname{Mean}(z)$ the mean intensity, was previously used in the BC model as the fidelity term. The Coefficient of Variation $(\mathrm{CoV})$ is defined as

$$
\operatorname{CoV}^{2}=\frac{\operatorname{Var}(z)}{(\operatorname{Mean}(z))^{2}} .
$$

The CoV value is higher in areas where there are edges than in areas that are uniform $[17,21]$, hence a higher value indicates that the pixels belong to the edges and a small value indicates the pixels belong to a uniform region. Indeed, the properties of $\mathrm{CoV}[17,21]$ suggest that it can be used as a fitting term as well as a good region detector. The experimental results using the $\mathrm{CoV}$ as the fidelity term to modify the CV model show that the converged contour tends to be next to the initial contour. We use the $C o V$ to detect non-spurious objects near an initial contour. Consequently, the level set formulation for (3.1) becomes

$$
\begin{aligned}
F\left(\phi, c_{1}, c_{2}\right)= & \mu \int_{\Omega} d(x, y) g(|\nabla z|) \delta(\phi)|\nabla \phi| d x d y+\lambda_{1} \int_{\Omega} \frac{\left(z(x, y)-c_{1}\right)^{2}}{c_{1}^{2}} H(\phi) d x d y \\
& +\lambda_{2} \int_{\Omega} \frac{\left(z(x, y)-c_{2}\right)^{2}}{c_{2}^{2}}(1-H(\phi)) d x d y
\end{aligned}
$$

and a modified minimisation problem from using $H_{\epsilon}$ and $\delta_{\epsilon}$ is

$$
\min _{\phi, c_{1}, c_{2}} F_{\epsilon}\left(\phi, c_{1}, c_{2}\right)
$$

where

$$
\begin{aligned}
F_{\epsilon}\left(\phi, c_{1}, c_{2}\right)= & \mu \int_{\Omega} d(x, y) g(|\nabla z|) \delta_{\epsilon}(\phi)|\nabla \phi| d x d y+\lambda_{1} \int_{\Omega} \frac{\left(z(x, y)-c_{1}\right)^{2}}{c_{1}^{2}} H_{\epsilon}(\phi) d x d y \\
& +\lambda_{2} \int_{\Omega} \frac{\left(z(x, y)-c_{2}\right)^{2}}{c_{2}^{2}}\left(1-H_{\epsilon}(\phi)\right) d x d y .
\end{aligned}
$$

Keeping $\phi$ fixed and minimising $F_{\epsilon}\left(\phi, c_{1}, c_{2}\right)$ with respect to $c_{1}$ and $c_{2}$, we have

$$
c_{1}(\phi)=\frac{\int_{\Omega} z^{2}(x, y) H_{\epsilon}(\phi) d x d y}{\int_{\Omega} z(x, y) H_{\epsilon}(\phi) d x d y}
$$

and

$$
c_{2}(\phi)=\frac{\int_{\Omega} z^{2}(x, y)\left(1-H_{\epsilon}(\phi)\right) d x d y}{\int_{\Omega} z(x, y)\left(1-H_{\epsilon}(\phi)\right) d x d y}
$$


Now keeping $c_{1}, c_{2}$ fixed and minimising $F_{\epsilon}$ with respect to $\phi$ yields the following EulerLagrange equation for $\phi$ :

$$
\begin{cases}\delta_{\epsilon}(\phi)\left[\mu \operatorname{div}\left(G(x, y) \frac{\nabla \phi}{|\nabla \phi|}\right)\right. & \\ \left.-\lambda_{1} \frac{\left(z(x, y)-c_{1}\right)^{2}}{c_{1}^{2}}+\lambda_{2} \frac{\left(z(x, y)-c_{2}\right)^{2}}{c_{2}^{2}}\right]=0 & \text { in } \Omega, \\ \frac{G(x, y) \delta_{\epsilon}(\phi)}{|\nabla \phi|} \frac{\partial \phi}{\partial \vec{n}}=0 & \text { on } \partial \Omega,\end{cases}
$$

where $G(x, y)=d(x, y) g(|\nabla z|)$.

Remark 3.1. Denominators in (3.1) and (3.2) can be zero in situations where we need to segment an object or region having zero value. For such cases, we can obtain the values of $c_{1}$ and $c_{2}$ as follows:

$$
c_{1}(\phi)=\frac{\int_{\Omega} z^{2}(x, y) H_{\epsilon}(\phi) d x d y}{\int_{\Omega} z(x, y) H_{\epsilon}(\phi) d x d y+\gamma}, \quad c_{2}(\phi)=\frac{\int_{\Omega} z^{2}(x, y)\left(1-H_{\epsilon}(\phi)\right) d x d y}{\int_{\Omega} z(x, y)\left(1-H_{\epsilon}(\phi)\right) d x d y+\gamma},
$$

where $\gamma$ is a small positive real number. Similarly, we may replace $c_{l}^{2}$ by $c_{l}^{2}+\gamma$ in (3.3).

We now add a balloon term $\alpha G(x, y)|\nabla \phi|$ to speed up the convergence of the evolution equation as in Ref. [3]. Thus we get

$$
\begin{aligned}
& \frac{\partial \phi}{\partial t}=\delta_{\epsilon}(\phi)\left[\mu \nabla \cdot\left(G(x, y) \frac{\nabla \phi}{|\nabla \phi|}\right)-\lambda_{1} \frac{\left(z(x, y)-c_{1}\right)^{2}}{c_{1}^{2}}\right. \\
& \left.\quad+\lambda_{2} \frac{\left(z(x, y)-c_{2}\right)^{2}}{c_{2}^{2}}+\alpha G(x, y)|\nabla \phi|\right] \text { in } \Omega, \\
& \phi(t, x, y)=\phi_{0}(x, y) \text { in } \Omega .
\end{aligned}
$$

The existence of $\phi$ can be proved along similar lines to Ref. [11].

\section{Numerical Methods}

We present two numerical methods for solving the nonlinear parabolic PDE (3.4).

\subsection{Semi-implicit method}

First we write the PDE (3.4) in a self-adjoint form

$$
\frac{\partial \phi}{\partial t}=\mu \delta_{\epsilon}(\phi) \nabla\left(G(x, y) \frac{\nabla \phi}{|\nabla \phi|}\right)+f(x, y)
$$

i.e.

$$
\frac{\partial \phi}{\partial t}=\mu \delta_{\epsilon}(\phi(x, y))\left[G(x, y) \nabla \cdot\left(\frac{\nabla \phi}{|\nabla \phi|}\right)+\nabla G(x, y) \cdot\left(\frac{\nabla \phi}{|\nabla \phi|}\right)\right]+f(x, y),
$$


where

$$
f(x, y)=\mu \delta_{\epsilon}(\phi)\left[-\lambda_{1} \frac{\left(z(x, y)-c_{1}\right)^{2}}{c_{1}^{2}}+\lambda_{2} \frac{\left(z(x, y)-c_{2}\right)^{2}}{c_{2}^{2}}\right]+\alpha G(x, y)|\nabla \phi| .
$$

Now using the differences given by

$$
\begin{array}{ll}
\Delta_{-}^{x}\left(\phi_{i, j}\right)=\phi_{i, j}-\phi_{i-1, j}, & \Delta_{+}^{x}\left(\phi_{i, j}\right)=\phi_{i+1, j}-\phi_{i, j}, \\
\Delta_{-}^{y}\left(\phi_{i, j}\right)=\phi_{i, j}-\phi_{i, j-1}, & \Delta_{+}^{y}\left(\phi_{i, j}\right)=\phi_{i, j+1}-\phi_{i, j}
\end{array}
$$

and a semi-implicit scheme, the discretised form of the above equation is

$$
\begin{aligned}
\frac{\phi_{i, j}^{n+1}-\phi_{i, j}^{n}}{\triangle t}= & \mu \delta_{\epsilon}\left(\phi_{i, j}^{n}\right) G_{i, j}\left[\frac{1}{h_{1}^{2}} \triangle_{-}^{x}\left(\frac{\triangle_{+}^{x} \phi_{i, j}^{n+1}}{\sqrt{\left(\triangle_{+}^{x} \phi_{i, j}^{n} / h_{1}\right)^{2}+\left(\triangle_{+}^{y} \phi_{i, j}^{n} / h_{2}\right)^{2}}}\right)\right] \\
& +\mu \delta_{\epsilon}\left(\phi_{i, j}^{n}\right) G_{i, j} \frac{1}{h_{2}^{2}} \triangle_{-}^{y}\left(\frac{\triangle_{+}^{y} \phi_{i, j}^{n+1}}{\sqrt{\left(\triangle_{+}^{x} \phi_{i, j}^{n} / h_{1}\right)^{2}}+\left(\triangle_{+}^{y} \phi_{i, j}^{n} / h_{2}\right)^{2}}\right) \\
& +\mu \frac{\delta_{\epsilon}\left(\phi_{i, j}^{n}\right)}{\left|\nabla \phi_{i, j}^{n}\right|}\left(\frac{1}{h_{1}^{2}} \triangle_{+}^{x} G(x, y) \triangle_{+}^{x} \phi_{i, j}^{n+1}\right) \\
& +\frac{\mu \delta_{\epsilon}\left(\phi_{i, j}^{n}\right)}{\left|\nabla \phi_{i, j}^{n}\right|}\left(\frac{1}{h_{2}^{2}} \triangle_{+}^{y} G(x, y) \triangle_{+}^{y} \phi_{i, j}^{n+1}\right)+f_{i, j} .
\end{aligned}
$$

Using $h_{1}=h_{2}=1$ (i.e. absorb $h_{1}$ and $h_{2}$ into the parameter $\mu$ ), we get

$$
\begin{aligned}
\frac{\phi_{i, j}^{n+1}-\phi_{i, j}^{n}}{\triangle t}= & \mu \delta_{\epsilon}\left(\phi_{i, j}^{n}\right) G_{i, j}\left[\left(\phi_{i+1, j}^{n+1}-\phi_{i, j}^{n+1}\right) C_{i, j}-\left(\phi_{i, j}^{n+1}-\phi_{i-1, j}^{n+1}\right) C_{i-1, j}\right. \\
& \left.+\left(\phi_{i, j+1}^{n+1}-\phi_{i, j}^{n+1}\right) C_{i, j}-\left(\phi_{i, j}^{n+1}-\phi_{i, j-1}^{n+1}\right) C_{i, j-1}\right] \\
& +\frac{\mu \delta_{\epsilon}\left(\phi_{i, j}^{n}\right)}{\left|\nabla \phi_{i, j}^{n}\right|}\left[\triangle_{+}^{x} G(x, y)\left(\phi_{i+1, j}^{n+1}-\phi_{i, j}^{n+1}\right)+\triangle_{+}^{y} G(x, y)\left(\phi_{i, j+1}^{n+1}-\phi_{i, j}^{n+1}\right)\right] \\
& +f_{i, j},
\end{aligned}
$$

where

$$
\begin{aligned}
& C_{i, j}=\frac{1}{\sqrt{\left(\Delta_{+}^{x} \phi_{i, j}^{n}\right)^{2}+\left(\Delta_{+}^{y} \phi_{i, j}^{n}\right)^{2}}}, \quad C_{i-1, j}=\frac{1}{\sqrt{\left(\Delta_{+}^{x} \phi_{i-1, j}^{n}\right)^{2}+\left(\Delta_{+}^{y} \phi_{i-1, j}^{n}\right)^{2}}}, \\
& C_{i, j-1}=\frac{1}{\sqrt{\left(\Delta_{+}^{x} \phi_{i, j-1}^{n}\right)^{2}+\left(\Delta_{+}^{y} \phi_{i, j-1}^{n}\right)^{2}}} .
\end{aligned}
$$

As the coefficients $C_{i-1, j}, C_{i, j}$ and $C_{i, j-1}$ are "frozen" at the $\mathrm{n}^{\text {th }}$ iteration, Eq. (4.2) defines a linear system of equations that can be solved by an iterative method. To speed the solution, we next develop an additive operator splitting (AOS) method (cf. $[16,25,26])$ to solve the PDE (3.4). 


\subsection{An additive operator splitting method}

The above semi-implicit method, although stable with respect to $\Delta t$, can be expensive to apply for spatial dimension $\geq 2$. Related to the famous ADI (alternating direction implicit methods [9]), the AOS scheme $[16,25]$ splits the two-dimensional spatial operator into a sum of two one-dimensional space discretiziations, so that the resulting linear system can be solved efficiently by applying the Thomas algorithm twice. (Other splitting methods, such as the multiplicative type, may also be considered.) We first rewrite the PDE (4.1) in the form

$$
\begin{aligned}
\frac{\partial \phi}{\partial t} & =\mu \delta_{\epsilon}(\phi) \nabla(F \nabla \phi)+f \\
& =\mu \delta_{\epsilon}(\phi)\left(\partial_{x}\left(F \partial_{x} \phi\right)+\partial_{y}\left(F \partial_{y} \phi\right)\right)+f
\end{aligned}
$$

where $F=G /|\nabla \phi|$, and consider the one-dimensional problem in the $x$-direction:

$$
\frac{\phi_{i, j}^{n+1}-\phi_{i, j}^{n}}{\Delta t}=\mu \delta_{\epsilon}(\phi)\left(F_{i+1 / 2, j}^{n}\left(\phi_{i+1, j}^{n+1}-\phi_{i, j}^{n+1}\right)-F_{i-1 / 2, j}^{n}\left(\phi_{i, j}^{n+1}-\phi_{i-1, j}^{n+1}\right)\right)+f_{i, j},
$$

i.e.

$$
\phi_{i, j}^{n+1}=\phi_{i, j}^{n}+\mu \Delta t\left(c_{1} \phi_{i+1, j}^{n+1}-c_{2} \phi_{i, j}^{n+1}+c_{3} \phi_{i-1, j}^{n+1}\right)+f_{i, j}
$$

where

$$
\begin{aligned}
& c_{1}=\delta_{\epsilon}(\phi) \frac{F_{i, j}^{n}+F_{i+1, j}^{n}}{2}, \quad c_{2}=\delta_{\epsilon}(\phi) \frac{F_{i-1, j}^{n}+2 F_{i, j}^{n}+F_{i+1, j}^{n}}{2}, \\
& c_{2}=\delta_{\epsilon}(\phi) \frac{F_{i, j}^{n}+F_{i-1, j}^{n}}{2} .
\end{aligned}
$$

After we solve the system of equations (4.3), we solve a similar system in the $y$-direction, before averaging the two solutions to get

$$
\left(I-2 \Delta t A_{l}\left(\Phi^{n}\right)\right) \Phi_{l}^{n+1}=f^{n} \quad \text { for } l=1,2
$$

and

$$
\Phi_{l}^{n+1}=\frac{1}{2} \sum_{l=1}^{2} \Phi_{l}^{n+1},
$$

where $I$ is the identity matrix and $A_{l}$ for $l=1,2$ is a tridiagonal matrix. The AOS scheme uses a 1-D semi-implicit scheme in spatial directions independently, so it is absolutely stable, and both computational effort and storage is linear in the number of pixels. Other methods for the solution of this PDE include the Additive-Multiplicative Operator Splitting (AMOS) schemes [4] and Multigrid methods. 


\section{Experimental Results}

Some simulation results are now given to show that the new CSM method preserves advantages of the BC method - such as robustness in terms of number of iterations, CPU time, segmenting noisy images. It has also been found that the CSM performs better when segmenting images with fuzzy edges, or with overlapping homogeneous regions. For the sake of more comprehensive comparisons, we also included the Gout model $[10,11]$ in the numerical experiments. For brevity, we shall denote by

M-1 - the Gout model,

M-2 - the BC model, and

M-3 - the proposed CSM.

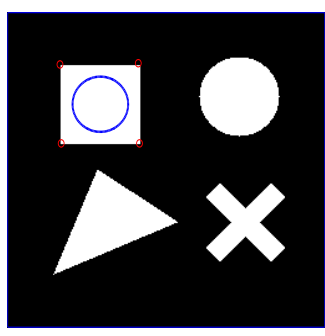

(a) Initial Contour

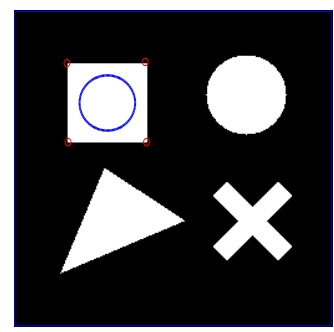

(e) Initial Contour

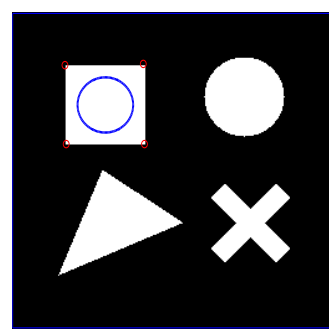

(i) Initial Contour

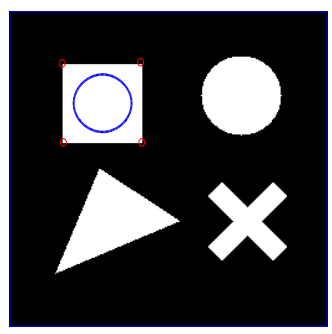

(b) 2 iterations of M-1

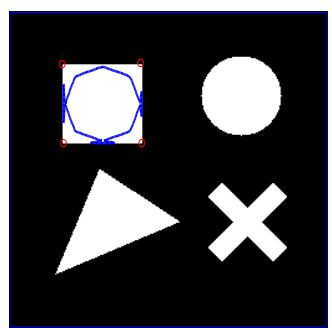

(f) 2 iterations of M-2

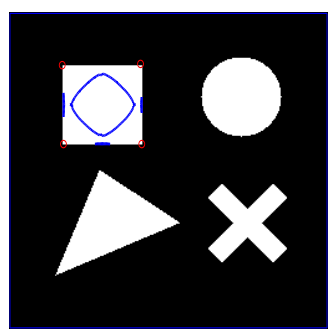

(j) 2 iterations of M-3

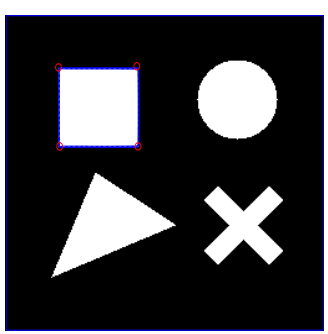

(c) After 45 iterations

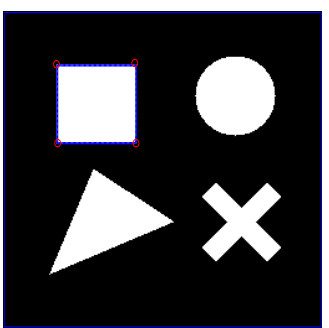

(g) After 10 iterations

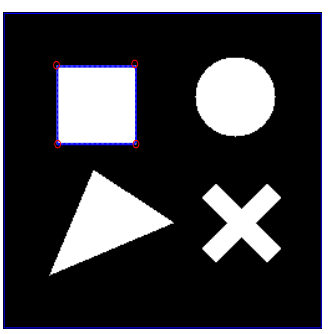

(k) After 10 iterations

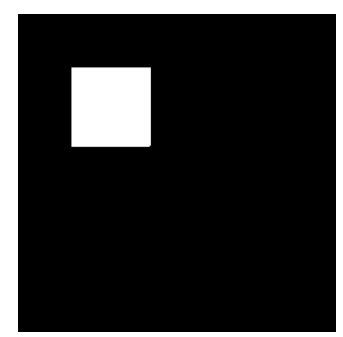

(d) Segmented Result

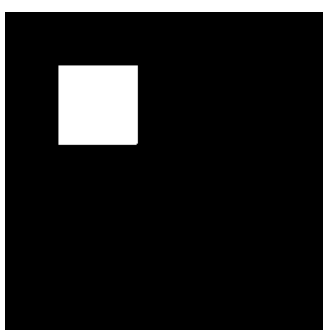

(h) Segmented Result

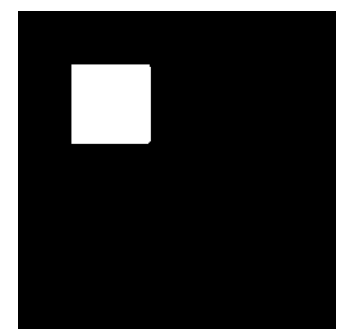

(1) Segmented Result

Figure 1: Simple example of segmenting a synthetic image, where $\mathrm{M}-1, \mathrm{M}-2$ and $\mathrm{M}-3$ have completed the task. M-1 took 19 seconds and both $\mathrm{M}-2$ and $\mathrm{M}-3$ took 4 seconds. For $\mathrm{M}-2$ and $\mathrm{M}-3$ the model parameters used are $\lambda=1$ and $\mu=256^{2} / 400$; and for all the three models $\alpha=-0.1$. The performance of model M-1, M-2 and M-3 can be seen in the first, second and third row, respectively. 
Below we present the original images, along with the results to compare these three methods. We begin with some simple examples where all three (M-1, M-2 and M-3) work well, and then give more examples that show M-3 has far better performance than both M-1 and M-2. In M-2 and M-3, we use $\lambda_{1}=\lambda_{2}=\lambda$. In each of the following figures, the first row shows the performance of M-1, the second row the performance of M-2, and the third row the performance of M-3. The first column shows the original image with initial contours, the second column displays the intermediate situations of the active contour of each model, the third column reveals the final solution in each model, and the fourth column displays the segmented version of the final solution for convenient deep observations.

The behaviour of M-2 can be seen in Figs. 2(g), 3(g), 4(g) and 5(g)-8(g). The experiments largely reveal the unpredictable behaviour of $\mathrm{M}-1$, as it mainly depends on edge

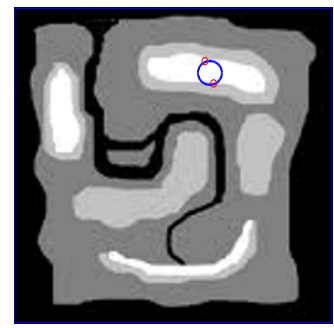

(a) Initial Contour

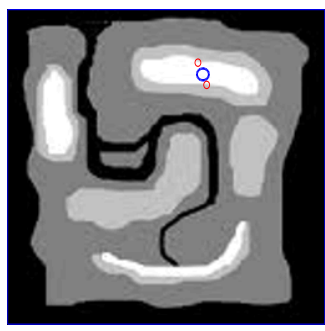

(e) Initial Contour

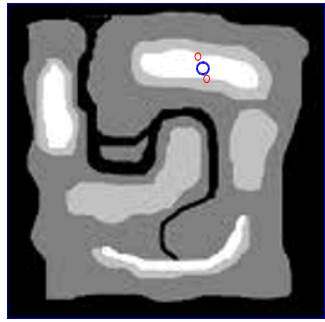

(i) Initial Contour

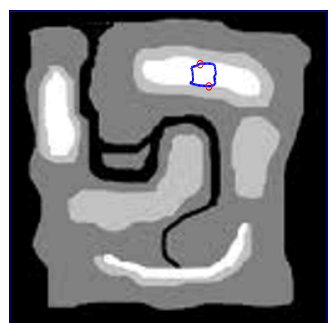

(b) 20 iterations of $\mathrm{M}-1$

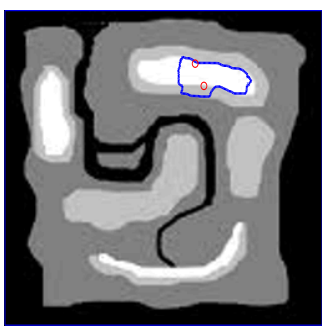

(f) 50 iterations of M-2

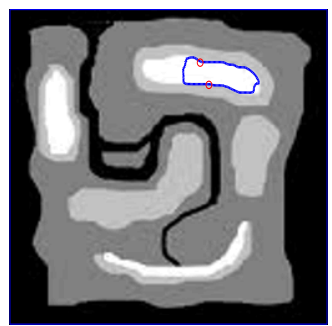

(j) 50 iterations of M-3

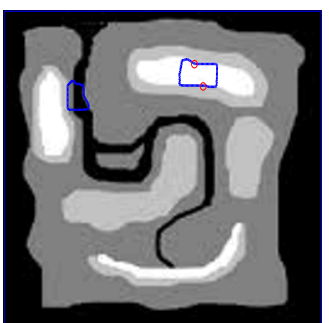

(c) After 10000 iterations

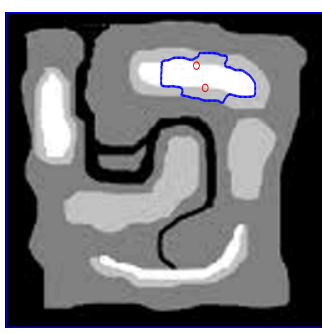

(g) After 200 iterations

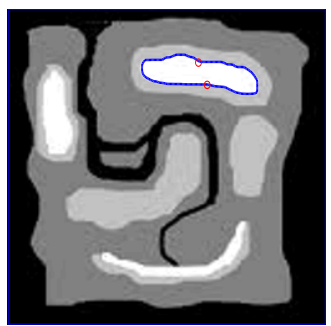

(k) After 200 iterations

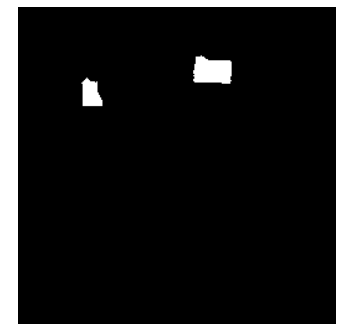

(d) M-1 Result

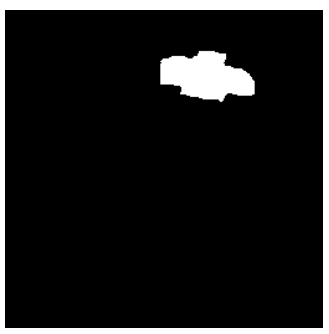

(h) M-2 Result

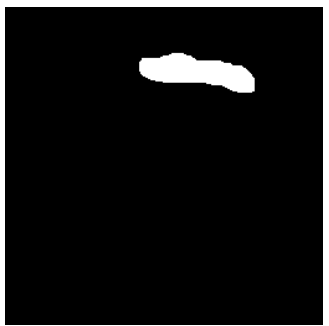

(1) M-3 Result

Figure 2: A comparison of $M-1, M-2$ and the proposed $M-3$ on segmenting a synthetic image. M-3 successfully detected the selected region. For M-3, the model parameters used are $\lambda=20, \mu=256^{2} / 5000$ and $\alpha=0$. Figs. 2(c), 2(d), 2(g) and 2(h) show that $M-1$ and $M-2$ are unable to detect the selected object in the given image. 


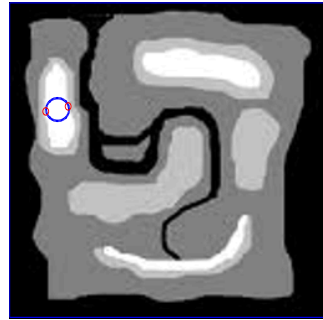

(a) Initial Contour

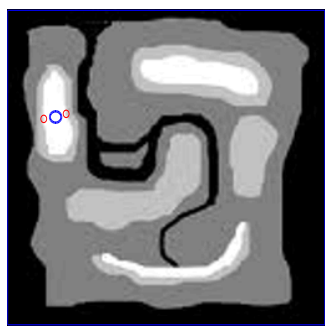

(e) Initial Contour

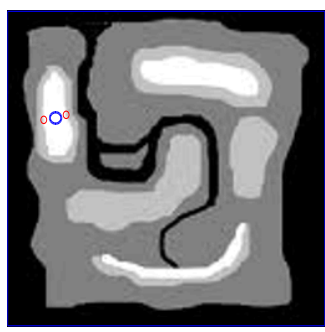

(i) Initial Contour

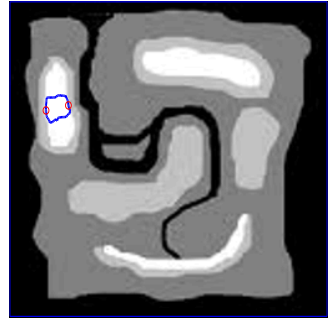

(b) 20 iterations of M-1

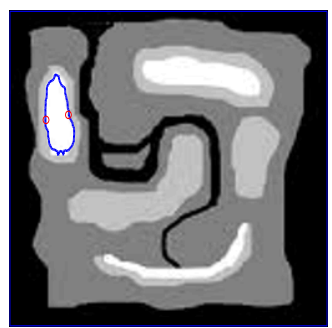

(f) 30 iterations of M-2

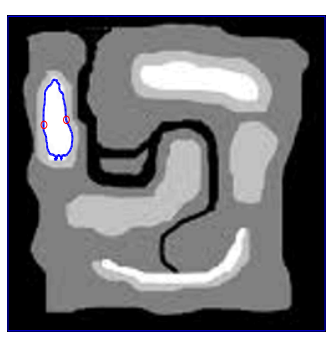

(j) 20 iterations of M-3

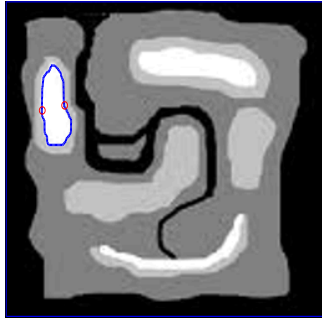

(c) After 1200 iterations

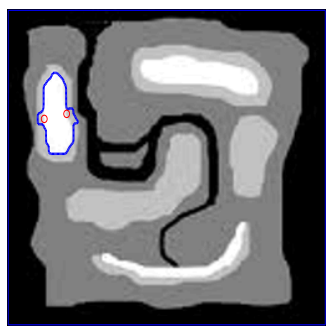

(g) After 200 iterations

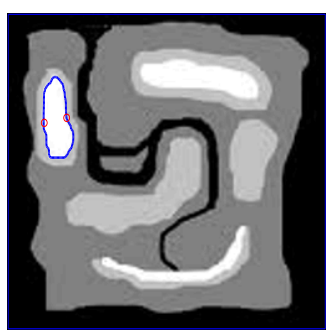

(k) After 35 iterations

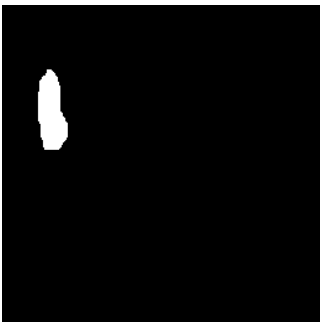

(d) M-1 Result

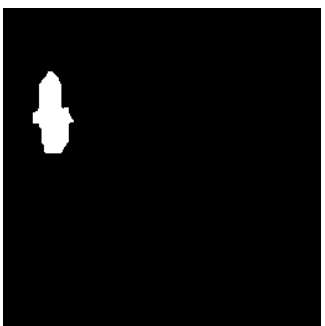

(h) M-2 Result

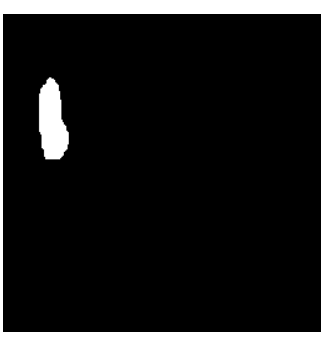

(1) M-3 Result

Figure 3: Model M-1 in figure 3(c) took 1200 iterations to converge to a final solution, whereas M-3 in Fig. 3(k) converged in only 35 iterations. Figs. $3(\mathrm{~g})$ and $3(\mathrm{~h})$ compare $\mathrm{M}-2$ (failing this example) and $\mathrm{M}-3$, where the model parameters used are $\lambda=15, \mu=256^{2} / 5000$ and $\alpha=0$.

detector function only. Figs. 2(c), 4(c) and 6(c) show that M-1 starts to detect other undesired regions or objects, instead of completing its selective segmentation task. In contrast with $\mathrm{M}-1$ and $\mathrm{M}-2$, the better performance of $\mathrm{M}-3$ can be seen. The experiments also show that M-3 is best in accurate and fast detection. and its success with images where M-1 and M-2 do not work.

In Fig. 2, the original image with initial contour is given. It is clear from Figs. 2(c), 2(d), 2(g) and 2(h) that M-1 and M-2 are unable to detect the region of interest. On the other hand, the success of M-3 can be seen in Figs. 2(k) and 2(l). In Fig. 2(a), the original image with initial contour is displayed. Fig. 2(c) shows that M-1 detected the selected region in 1200 iterations, whereas Fig. 2(k) shows that M-3 detected the same region in only 35 iterations. On the other hand, the performance of M-2 can easily be interpreted in 


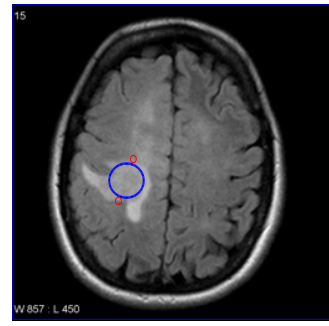

(a) Initial Contour

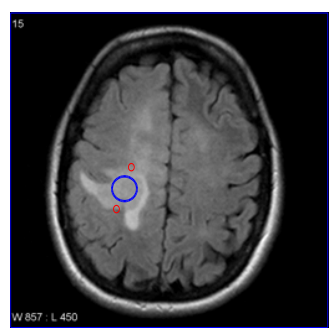

(e) Initial Contour

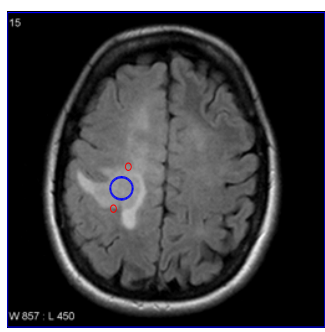

(i) Initial Contour

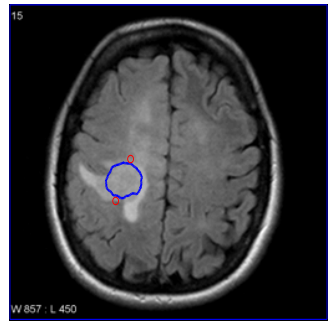

(b) 20 iterations of M-1

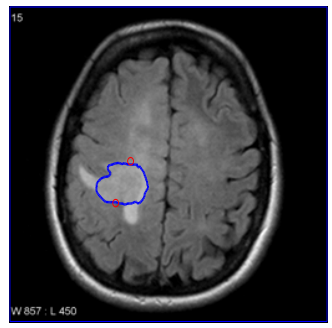

(f) 50 iterations of $\mathrm{M}-2$

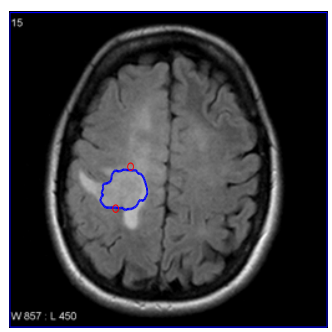

(j) 50 iterations of M-3

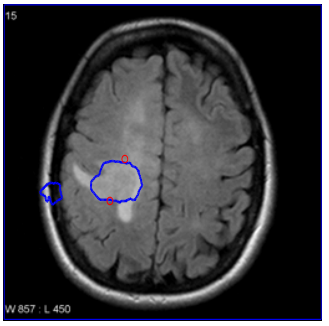

(c) After 5000 iterations

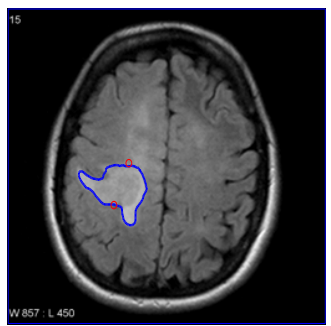

(g) After 800 iterations

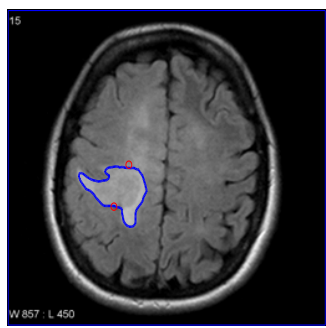

(k) After 800 iterations

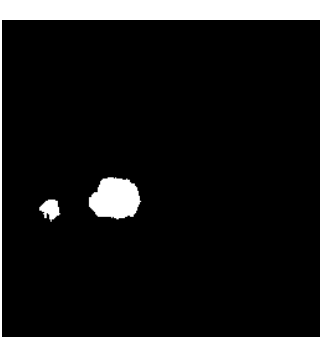

(d) Segmented Result

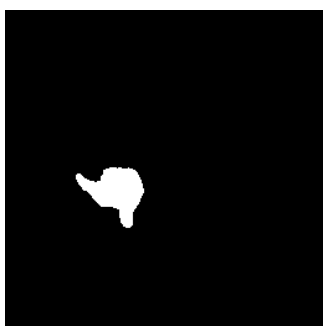

(h) Segmented Result

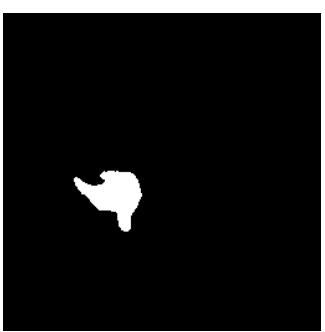

(1) Segmented Result

Figure 4: The quality of detection of M-2 and M-3 can readily be compared from Figs. 4(g), 4(h), 4(k) and $4(\mathrm{I})$. Fig. 4 (c) reveals that $M-1$ (failing this example) is unable to complete the task. For $M-3$, the parameters used are $\lambda=4, \mu=256^{2} / 10000$ and $\alpha=0$.

Fig. 2(g). In Fig. 4(a), a medical image with initial contour is displayed. The accuracy of detection of M-2 and M-3 is clearly visible in Figs. 4(g), 4(h), 4(k) and 4(l).

Fig. 5 exhibits a real breast image, in which we wish to detect a white region. Although It can be seen in Figs. 5(c) and 5(d) that M-1 detected the desired region, it is notable that it took 800 iterations to search for an ideal initial contour for this model. An ideal initial contour for M-1 is a contour to be selected almost on the boundary of an object or region of interest. It can be seen clearly in Figs. 2(a), 3(a), 5(a), 6(a) and 7(a) that an ideal initial contour is provided to $\mathrm{M}-1$, but still the results are devastating. In contrast, it can be observed in Figs. 2(i), 3(i), 4(i), 5(i), 6(i), 7(i), 8(i) and 9(i) that M-3 gives the best results without demanding an ideal initial contour. Fig. 6 displays a synthetic image and the performance of all of the three models. The proposed M-3 successfully detects a 


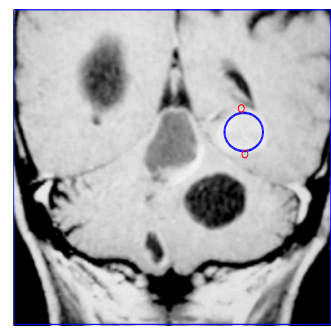

(a) Initial Contour

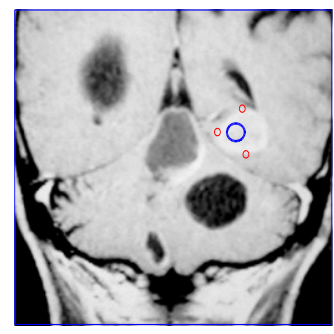

(e) Initial Contour

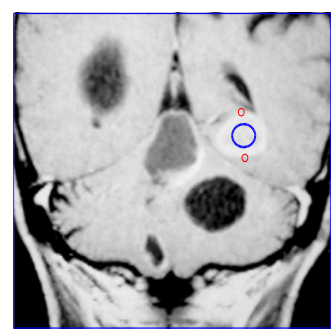

(i) Initial Contour

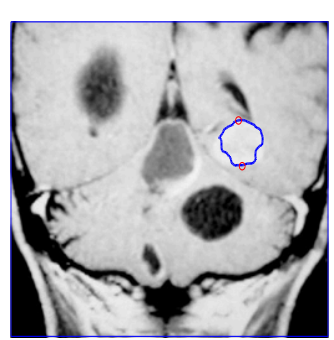

(b) 150 iterations of M-1

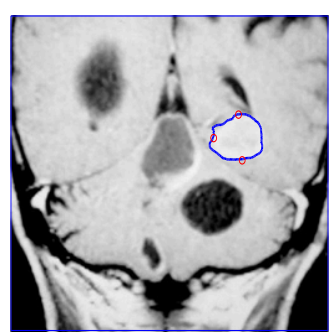

(f) 50 iterations of M-2

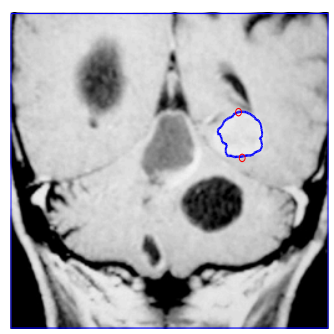

(j) 50 iterations of M-3

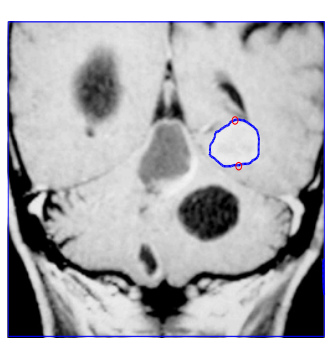

(c) After 800 iterations

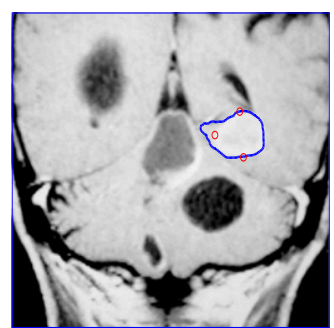

(g) After 500 iterations

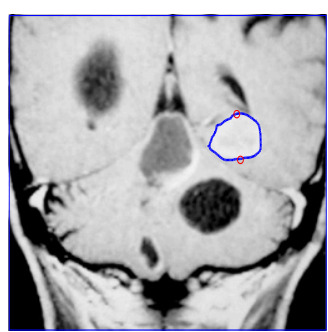

(k) After 380 iterations

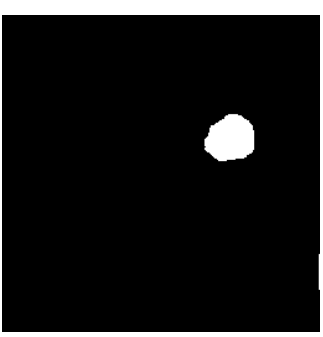

(d) Segmented Result

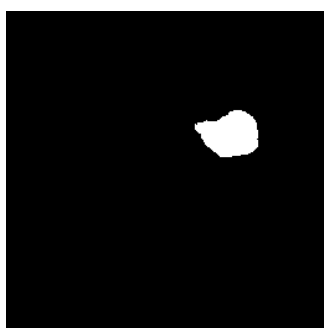

(h) Segmented Result

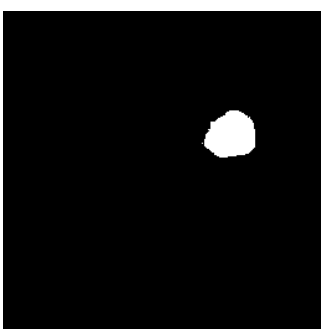

(l) Segmented Result

Figure 5: M-3 successfully detects a white spot in a real breast image. In Fig. 5(g), it is clear that the active contour of $\mathrm{M}-2$ has crossed the boundary of the region of interest. For $\mathrm{M}-3$, the parameters used are $\lambda=20, \mu=256^{2} / 5000$ and $\alpha=0$.

selected region in the synthetic image - cf. Fig, 6(k). Figs. 6(c) and 6(g) show that M-1 and $\mathrm{M}-2$ are unable to detect the selected object in the given image.

Fig. 7 exhibits a real eye image and demonstrates the performance of all three models by detecting a black region. Fig. 7(k) shows the successful detection by M-3 of the desired region, whereas Figs. $7(\mathrm{c})$ and $7(\mathrm{~g})$ show that M-1 and M-2 are unable to complete the task. A real heart image can viewed in Fig. 8. Successful detection of the selected portion in a real heart image by M-3 can be seen in Fig. 8(k), whereas Figs. 8(g) and 8(c) show the inaccurate detection by M-1 and M-2. In Fig. 9, a real abdominal image can be viewed. A comparison of Figs. 9(k), 9(g) and 9(c) shows that M-3 successfully treats that image, whereas M-1 and M-2 are unable to complete the task. Finally, Fig. 10 exhibits the performance of M-3 with fog images, which can be extended to video segmentation in many applications. 


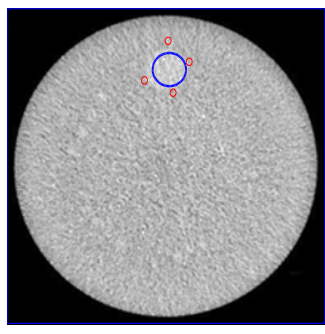

(a) Initial Contour

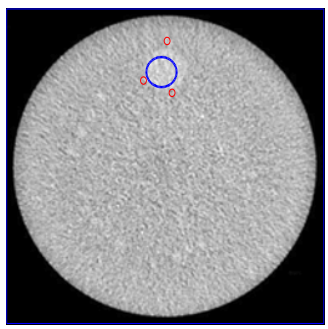

(e) Initial Contour

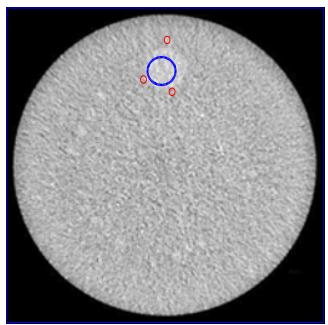

(i) Initial Contour

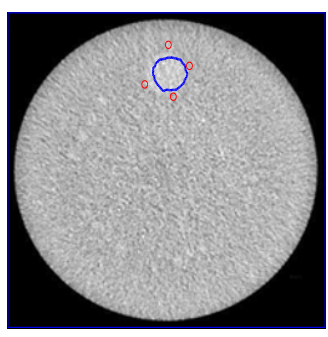

(b) 100 iterations of M-1

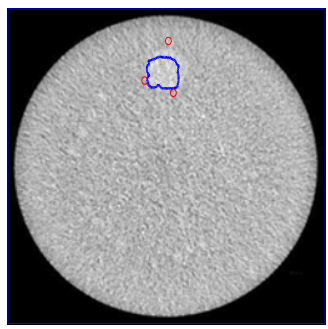

(f) 20 iterations of M-2

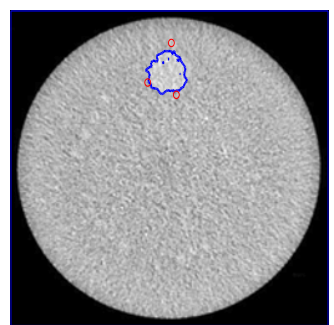

(j) 20 iterations of M-3

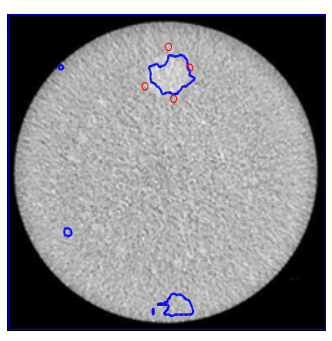

(c) After 5000 iterations

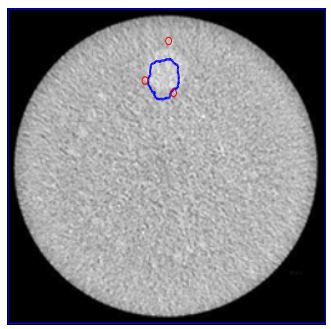

(g) After 370 iterations

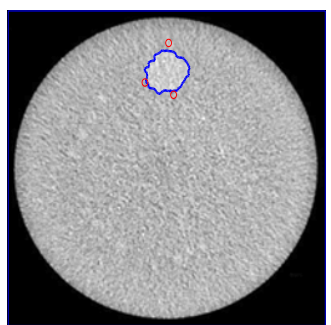

(k) After 400 iterations

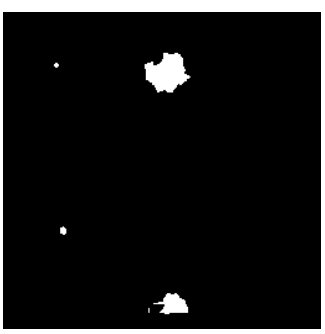

(d) Segmented Result

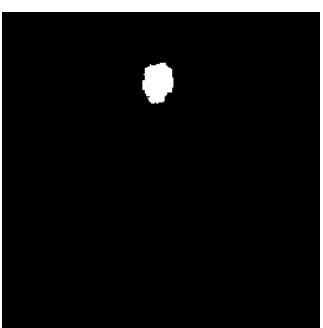

(h) Segmented Result

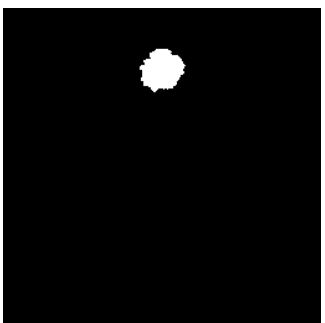

(1) Segmented Result

Figure 6: Successful detection of M-3 of a selected region in a synthetic image is shown in Fig. 6(k). Figs. $6(\mathrm{c}), 6(\mathrm{~g})$ show that $\mathrm{M}-1$ and $\mathrm{M}-2$ are unable to detect the selected object in the given image. For M-3, the parameters used are $\lambda=150, \mu=256^{2} / 4000$ and $\alpha=0$.

To summarize, in performing selective segmentation on challenging images with nearly equal intensity regions or fuzzy edges:

- the performance of M-2 is less effective because the active contour often crosses the boundary of an object of interest in the image, so is unable to detect the actual boundary and consequently the region of interest in the image; and

- the proposed new M-3 outperforms both M-1 and M-2.

\section{Conclusion}

A new active contour model for selective image segmentation based on coefficient of variation is presented, which performs better than previous models in segmenting images 


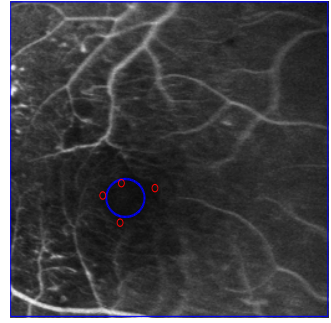

(a) Initial Contour

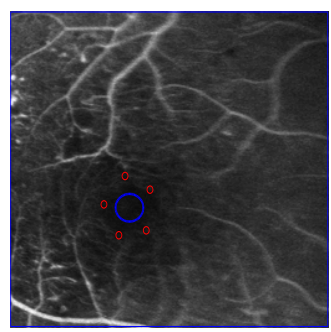

(e) Initial Contour

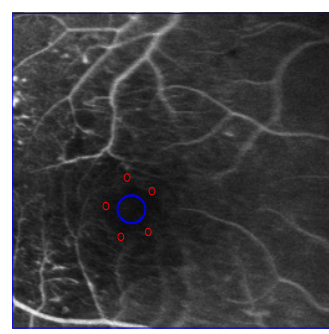

(i) Initial Contour

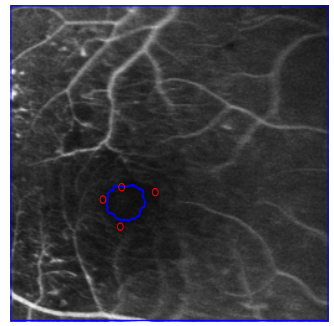

(b) 100 iterations of M-1

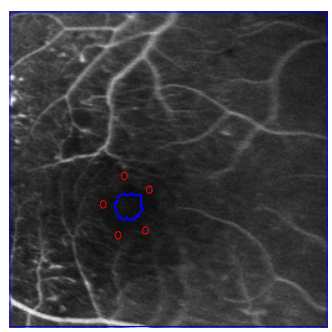

(f) 20 iterations of $\mathrm{M}-2$

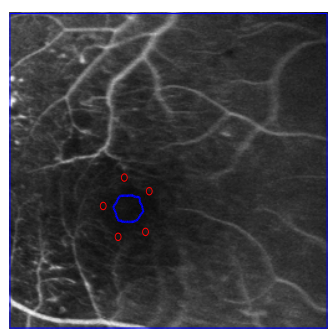

(j) 20 iterations of M-3

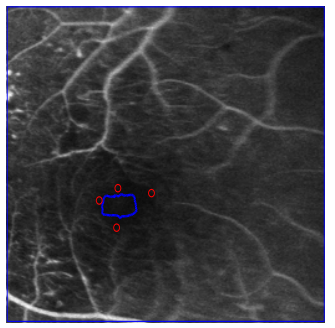

(c) After 10000 iterations

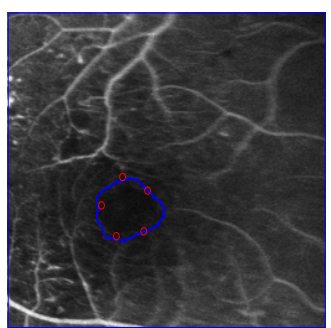

(g) After 1000 iterations

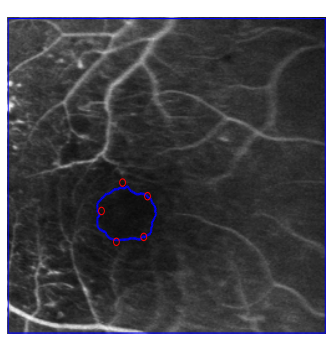

(k) After 1200 iterations

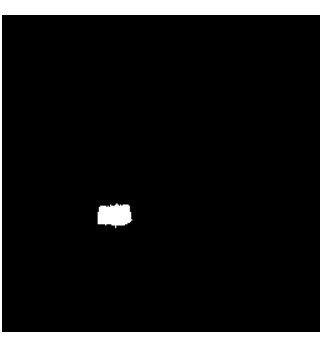

(d) Segmented Result

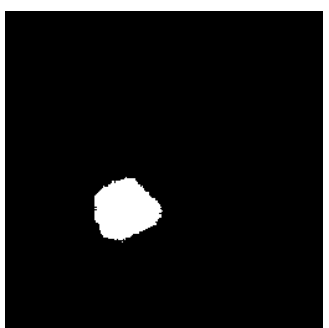

(h) Segmented Result

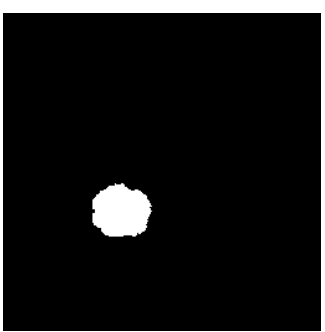

(1) Segmented Result

Figure 7: These results show the performance of all three models in detecting a black region in a real eye image. Fig. $7(\mathrm{k})$ shows the successful detection by $\mathrm{M}-3$, but in Figs. $7(\mathrm{c})$ and $7(\mathrm{~g}) \mathrm{M}-1$ and $\mathrm{M}-2$ are unable to complete the tasks. For $M-3$, the parameters used are $\lambda=0.4, \mu=256^{2} / 20000$ and $\alpha=-0.00125$.

with objects where edges are not prominent. The new model is able to detect objects with overlapping regions of homogeneous intensities, and it can also detect object with nonhomogeneous intensities. In future, we plan to develop fast multi-grid methods for the solution of differential equations arising from minimisation of the model, and to extend these results to segmenting 3-D images.

\section{References}

[1] S. E. Ahmed. A pooling methodology for coefficient of variation. Sankhya: The Indian Journal of Statistics, 57(B1):57-75, 1995.

[2] R. Adams and L. Bischof. Seeded region growing. IEEE Transactions on Pattern Analysis and Machine Intelligence, 16(6):641-647, 1994. 


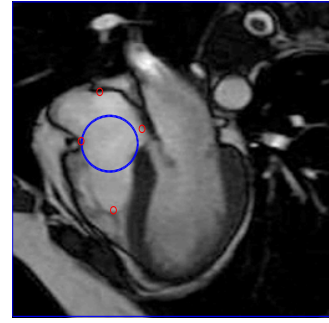

(a) Initial Contour

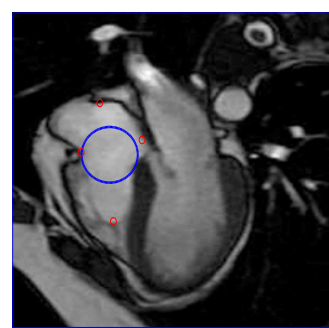

(e) Initial Contour

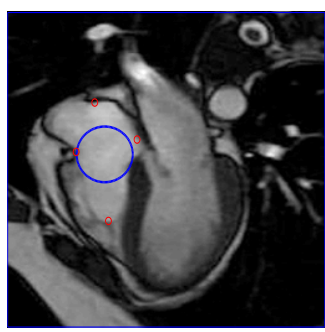

(i) Initial Contour

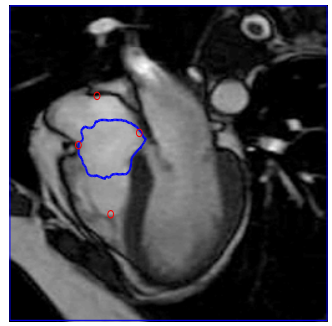

(b) 500 iterations of M-1

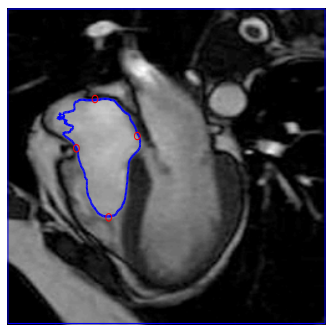

(f) 30 iterations of M-2

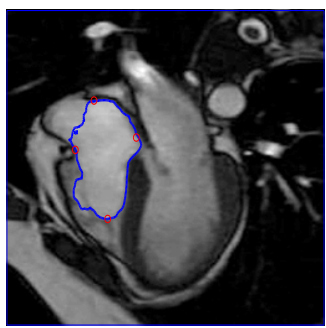

(j) 170 iterations of M-3

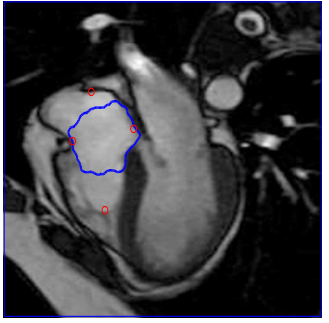

(c) After 10000 iterations

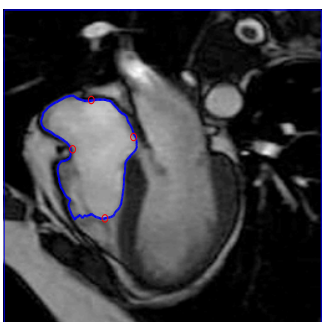

(g) After 500 iterations

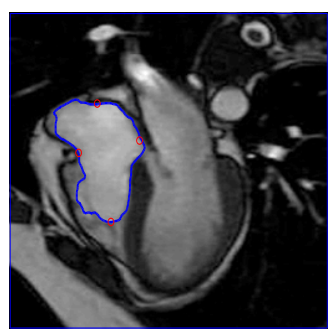

(k) After 340 iterations

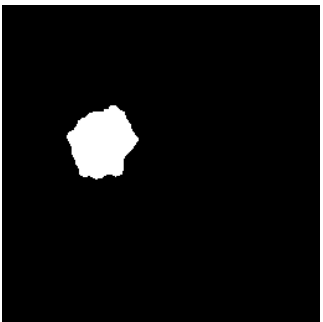

(d) Segmented Result

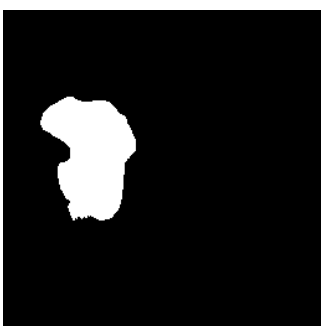

(h) Segmented Result

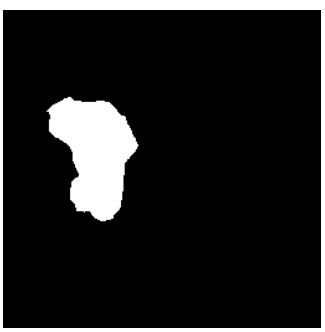

(l) Segmented Result

Figure 8: Successful detection of a selected portion of a real heart image by M-3 is shown in Fig. 8(k), where the parameters used are $\lambda=20, \mu=256^{2} / 5000$ and $\alpha=0$. In contrast, the uncompleted tasks by $\mathrm{M}-1$ and $\mathrm{M}-2$ can be seen clearly in Figs. $8(\mathrm{c})$ and $8(\mathrm{~g})$.

[3] N. Badshah and K. Chen. Image selective segmentation under geometrical constraints using an active contour approach. Mathematics of Computation, 7:759-778, 2010.

[4] D. Barash and R. Kimmel An accurate operator splitting scheme for nonlinear diffusion filtering. HP Laboratories Israel1 HPL-2000-48(R.1) August 1st, 2000

[5] V. Caselles, R. Kimmel, and G. Sapiro. Geodesic active contours. International Jornal of Computer Vision, 22(1):61-79, 1997.

[6] T. F. Chan and L. A. Vese. Active contours without edges. IEEE Transactions on Image Processing, 10(2):266-277, 2001.

[7] K. Chen. Matrix Preconditioning Techniques and Applications. Cambridge University Press, 2005.

[8] L. D. Cohen. On active contour models and balloons. Computer Vision, Graphics, and Image Processing: Image Understanding, 53(2):211-218, 1991.

[9] J. Douglas and H. H. Rachford. On the numerical solution of heat conduction problems in two and three space variables. Transactions of the American Mathematical Society, 82:421-439, 1956. 


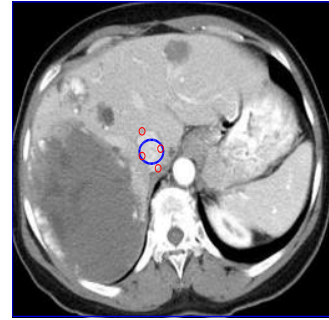

(a) Initial Contour

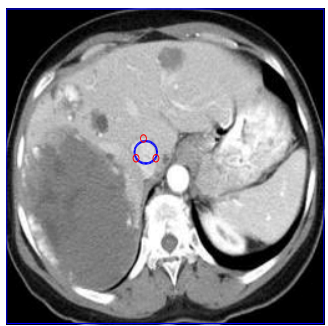

(e) Initial Contour

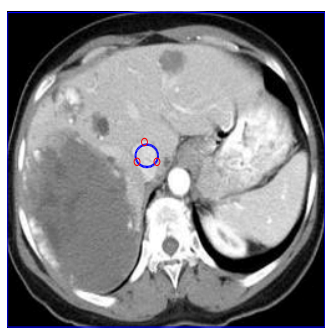

(i) Initial Contour

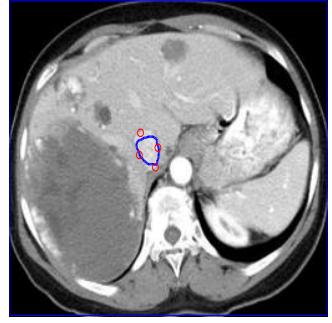

(b) 500 iterations of M-1

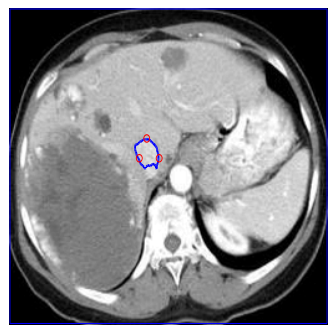

(f) 14 iterations of M-2

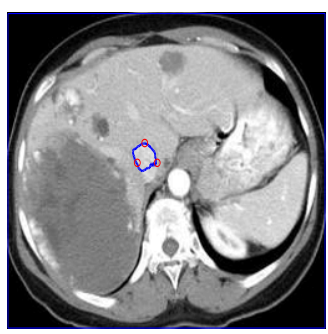

(j) 60 iterations of M-3

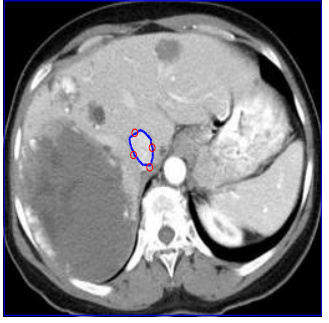

(c) After 10000 iterations

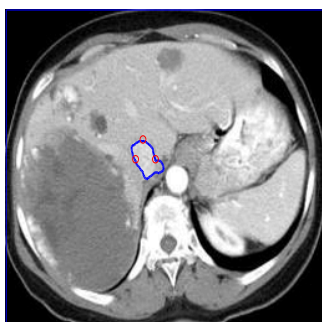

(g) After 370 iterations

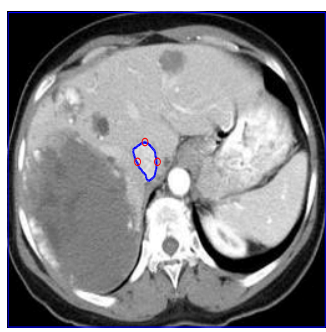

(k) After 135 iterations

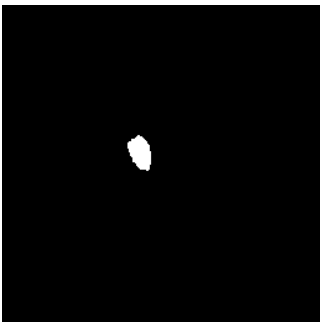

(d) Segmented Result

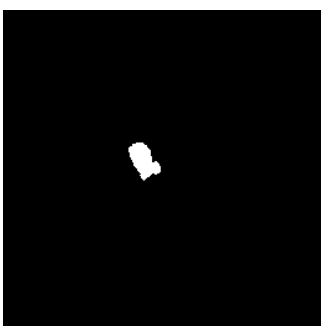

(h) Segmented Result

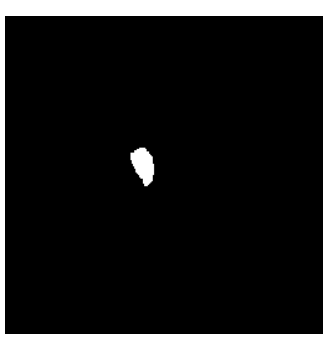

(l) Segmented Result

Figure 9: For a real abdominal image, the results from the three models are displayed. In Figs. 9(k), $9(\mathrm{~g})$ and $9(\mathrm{c})$, it can be seen that M-3 successfully completed the task, where the parameters used are $\lambda=2, \mu=256^{2} / 1500$ and $\alpha=-0.01$.

[10] C. Gout, C. L. Guyader, and L. A. Vese. Segmentation under geometrical conditions with geodesic active contour and interpolation using level set method. Numerical Algorithms, 39:155-173, 2005.

[11] C. L. Guyader and C. Gout. Geodesic active contour under geometrical conditions theory and 3D applications. Numerical Algorithms, 48:105-133, 2008.

[12] M. Jeon, M. Alexander, W. Pedrycz, and N. Pizzi. Unsupervised hierarchical image segmentation with level set and additive operator splitting. Pattern Recognition Letters, 26(10):1461$1469,2005$.

[13] M. Kass, A. Witkin, and D. Terzopoulos. Snakes: Active contours models. International Jornal of Computer Vision, 1:321-331, 1988.

[14] Y. Leclerc. Region growing using the MDL principle. In: DARPA Image Understanding Workshop, 1990.

[15] Y. B. Li and J. S. Kim. Multiphase image segmentation using a phase-field model. Computers and Mathematics with Applications, 62:737-745, 2011.

[16] T. Lu, P. Neittaanmaki, and X. C. Tai. A parallel splitting up method and its application to 


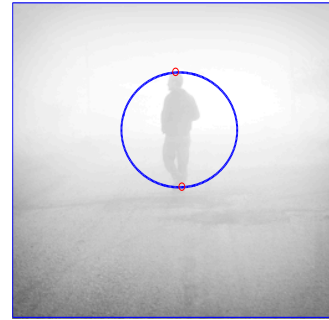

(a) Initial Contour

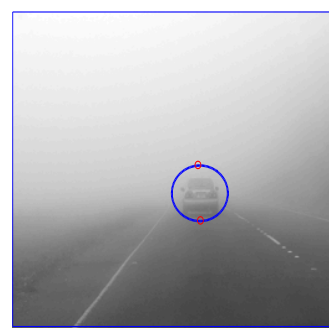

(e) Initial Contour

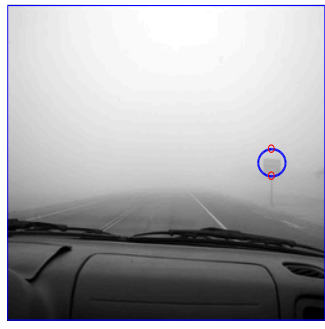

(i) Initial Contour

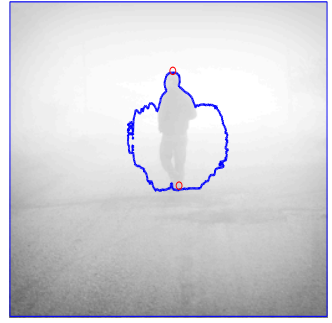

(b) After 20 iterations

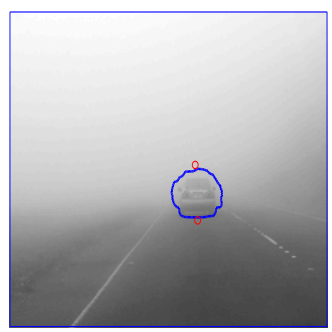

(f) After 25 iterations

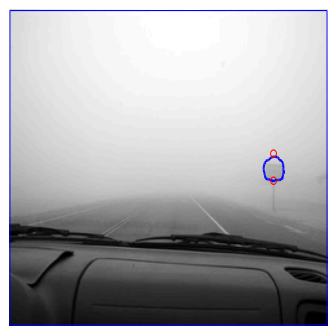

(j) After 2 iterations

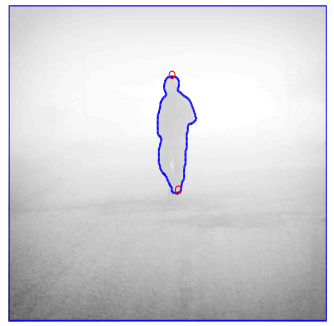

(c) After 115 iterations

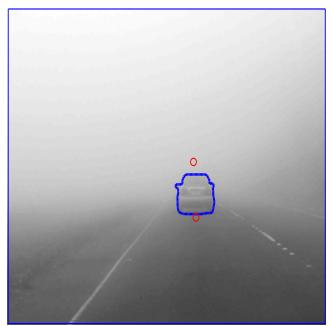

(g) After 65 iterations

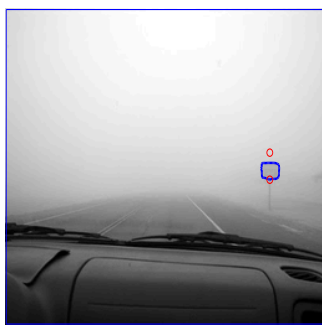

(k) After 10 iterations

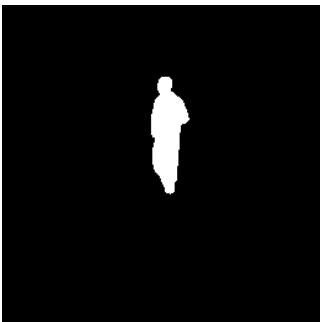

(d) Segmented Result

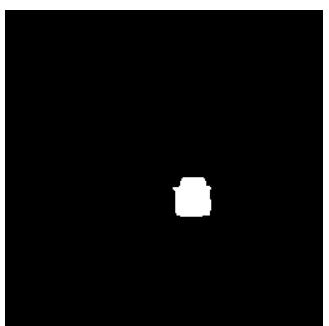

(h) Segmented Result

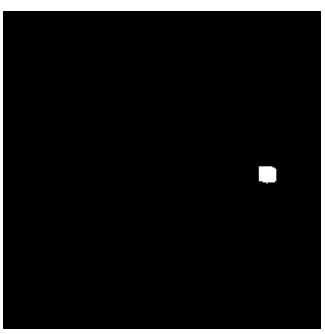

(1) Segmented Result

Figure 10: Performance of M-3 in fog images. For the foggy man image (c), the M-3 parameters used are: $\lambda=0.01, \mu=5$ and $\alpha=-0.00000151$; for $(\mathrm{g}), \lambda=0.01, \mu=0.5$ and $\alpha=0.00151$; and for $(\mathrm{k})$, $\lambda=0.5, \mu=2$ and $\alpha=-0.0007$.

Navier-Stokes equations. Applied Mathematics Letters, 4(2):25-29, 1991.

[17] M. Mora, C. Tauber, and H. Batatia. Robust level set for heart cavities detection in ultrasound images. Computers in Cardiology, 32:235-238, 2005.

[18] D. Mumford and J. Shah. Optimal approximation by piecewise smooth functions and associated variational problems. Communications on Pure Applied Mathematics, 42:577-685, 1989.

[19] S. Osher and R. Fedkiw. Level Set Methods and Dynamic Implicit Surfaces, Springer Verlag, 2003.

[20] S. Osher and J. A. Sethian. Fronts propagating with curvature-dependent speed: Algorithms based on Hamilton-Jacobi formulations. Journal of Computational Physics, 79(1):12-49, 1988.

[21] M. A. Schulze and Q. X. Wu. Nonlinear edge-preserving smoothing of synthetic aperture radar images. Proceedings of the New Zealand Image and Vision Computing '95 Workshop, pp. 65-70, 1995.

[22] J. A. Sethian. Level Set Methods and Fast Marching Methods: Evolving Interfaces in Com- 
putational Geometry, Fluid Mechanics, Computer Vision and Material Science, Cambridge University Press, 1999.

[23] L. A. Vese and T. F. Chan. A multiphase level set framework for image segmentation using the Mumford and Shah model. International Journal of Computer Vision, 50(3):271-293, 2002.

[24] L. Vincent and P. Soille. Watersheds in digital spaces - An efficient algorithm based on immersion. IEEE Transactions Pattern Analysis and Machine Learning, 13(6):583-598, 1994.

[25] J. Weickert, B. M. T. H. Romeny, and M. A. Viergever. Efficient and reliable schemes for nonlinear diffusion filtering. IEEE Transactions On Image Processing, 7:398-410, 1998.

[26] J. Weickert and G. Kühne. Fast methods for implicit active contours models. Geometric Level Set Methods in Imaging, Vision, and Graphics, Part II, 43-57, 2003.

[27] Y. Yu and S. T. Acton. Edge detection in ultrasound imagery using the instantaneous coefficient of variation. IEEE Transactions On Image Processing, 13(12):1640-1655, 2004. 